\title{
Prediction of margin involvement and local recurrence after skin-sparing and simple mastectomy
}

DOI:

10.1016/j.ejso.2016.04.055

\section{Document Version}

Accepted author manuscript

Link to publication record in Manchester Research Explorer

\section{Citation for published version (APA):}

Al-Himdani, S., Timbrell, S., Tan, K., Morris, J., \& Bundred, N. J. (2016). Prediction of margin involvement and local recurrence after skin-sparing and simple mastectomy. European Journal of Surgical Oncology, 42(7), 935-941. https://doi.org/10.1016/j.ejso.2016.04.055

\section{Published in:}

European Journal of Surgical Oncology

\section{Citing this paper}

Please note that where the full-text provided on Manchester Research Explorer is the Author Accepted Manuscript or Proof version this may differ from the final Published version. If citing, it is advised that you check and use the publisher's definitive version.

\section{General rights}

Copyright and moral rights for the publications made accessible in the Research Explorer are retained by the authors and/or other copyright owners and it is a condition of accessing publications that users recognise and abide by the legal requirements associated with these rights.

\section{Takedown policy}

If you believe that this document breaches copyright please refer to the University of Manchester's Takedown Procedures [http://man.ac.uk/04Y6Bo] or contact uml.scholarlycommunications@manchester.ac.uk providing relevant details, so we can investigate your claim.

\section{OPEN ACCESS}




\section{elsevier_YEJSO_4342}

\section{Prediction of margin involvement and local recurrence after skin-sparing and simple mastectomy}

\section{S. Al-Himdania}

S. Timbrell ${ }^{a}$

K.T. Tan

J. Morris

N.J. Bundreda,*

bundredn@manchester.ac.uk

aDepartment of Academic Breast Surgery, University Hospital of South Manchester NHS Foundation Trust, Manchester, UK

bDepartment of Medical Statistics, University Hospital of South Manchester NHS Foundation Trust, Manchester, UK

*Corresponding author. University Hospital of South Manchester, Southmoor Road, Manchester, M23 9LT, UK. Tel.: +44 (0)161 2915861.

\section{Abstract}

Skin-sparing mastectomy (SSM) facilitates immediate breast reconstruction. We investigated locoregional recurrence rates after SSM compared with simple mastectomy and the factors predicting oncological failure.

\section{Methods}

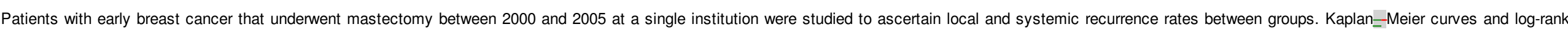
test were used to evaluate disease-free survival.

\section{Results}

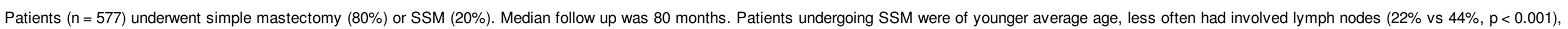

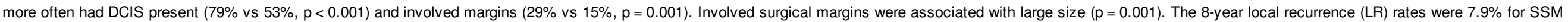

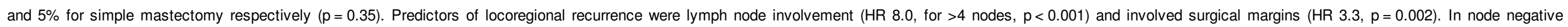
patients, SSM was a predictor of locoregional recurrence (HR $4.8[1.1,19.9], p=0.033)$.

Conclusion(s)

Delayed reconstruction is more appropriate for node positive early breast cancer after post-mastectomy radiotherapy. Re-excision of involved margins is essential to prevent local recurrence after mastectomy.

Keywords: Breast cancer; Local recurrence; Mastectomy; Breast reconstruction; Surgical margins; DCIS

\section{Introduction}

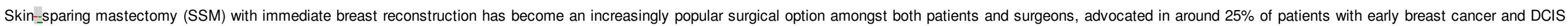

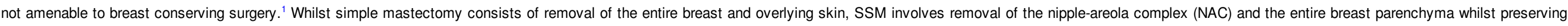
the native skin envelope. Patient satisfaction is improved as the preserved skin creates a more naturally appearing cosmetic result.

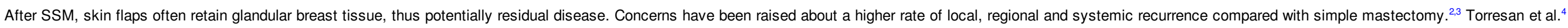

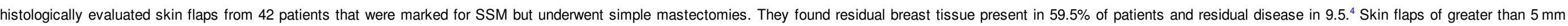

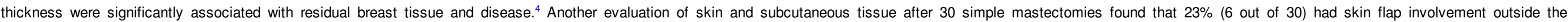


nipple__areolar complex (NAC). ${ }^{5}$

Skin tethering, large tumour size and perineural infiltration were all significant parameters associated with skin involvement. ${ }^{5}$

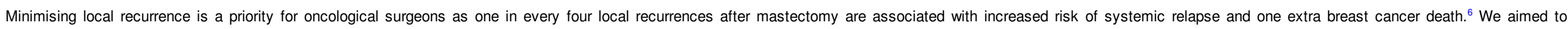
determine the factors predicting local recurrence after mastectomy.

\section{Materials and methods}

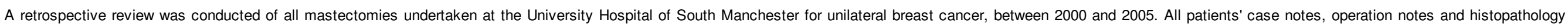

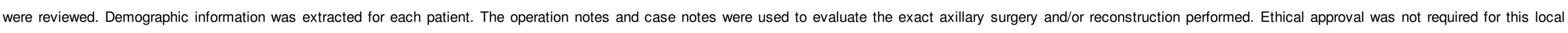
cancer audit in a single hospital unit.

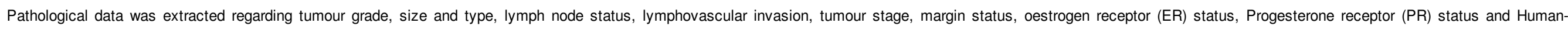
Epidermal growth factor-2 status (HER-2). Use of neoadjuvant chemotherapy, adjuvant chemotherapy/radiotherapy/hormone therapy was also evaluated.

Follow-up data evaluated included the time to local, regional, systemic recurrence and death. Inflammatory and locally advanced breast cancers were excluded from the study.

\section{Surgical technique}

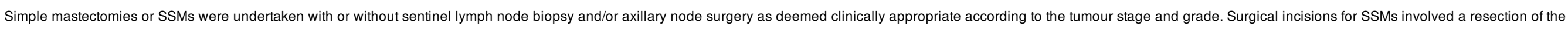

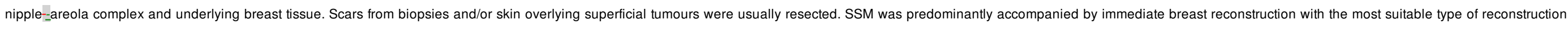

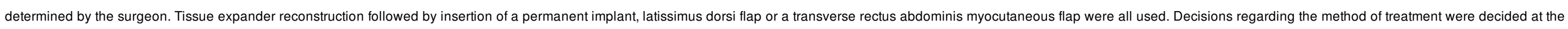
multi-disciplinary team meetings based on clinical examination, imaging and patient preference.

\section{Follow up and recurrence}

Patients were followed up annually by clinical examination of the breast, chest wall and axillary lymph nodes as well as mammography imaging.

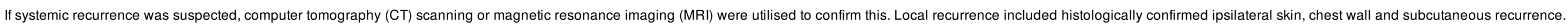
Regional recurrence included regional lymph node metastases.

\section{Statistical analysis}

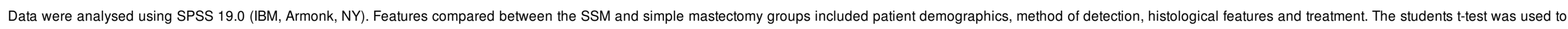

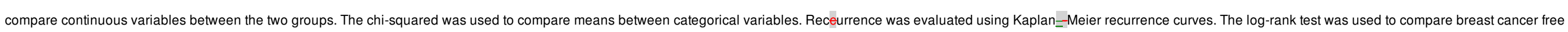
survival (recurrence) between the two groups. Factors associated with incomplete margins were identified by logistic regression analysis.

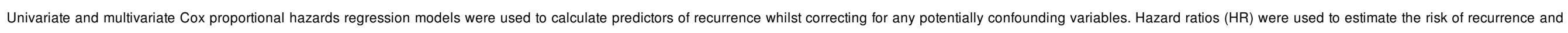
death, utilising a 95\% confidence interval (Cl). Statistical significance was considered when $\mathrm{P} \underline{\mathrm{p}} \leq 0.05$.

\section{Results}

\section{Patient demographics and histological features of tumour}

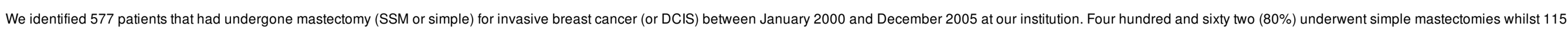

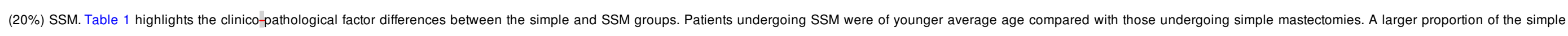
mastectomies were not screen-detected, had DCIS present and had lymphovascular invasion, compared with those in the SSM group. There was no difference in receptor status between groups. 


\section{elsevier_YEJSO_4342}

Table 1 Comparison simple and SSM groups: clinicopathological factors and recurrence.

\begin{tabular}{|c|c|c|c|}
\hline & Simple $(n=462)$ & $\operatorname{SSM}(n=115)$ & Comparison of groups \\
\hline Age (years) & $61.6(22-96)$ & $49.1(29-69)$ & $P<0.001^{a}$ \\
\hline Symptomatic & $314(68 \%)$ & $65(56 \%)$ & $P=0.028^{b}$ \\
\hline \multicolumn{4}{|l|}{ Grade } \\
\hline 0 & $11(2 \%)$ & $2(2 \%)$ & \multirow[t]{4}{*}{$P=0.12^{c}$} \\
\hline 1 & $30(6 \%)$ & $7(6 \%)$ & \\
\hline 2 & $176(38 \%)$ & $60(52 \%)$ & \\
\hline 3 & $245(53 \%)$ & $46(40 \%)$ & \\
\hline \multicolumn{4}{|l|}{ Tumour size $(n=548)$} \\
\hline$<15 \mathrm{~mm}$ & $98(22 \%)$ & $28(26 \%)$ & \multirow[t]{3}{*}{$P=0.02^{c}$} \\
\hline $15-25 \mathrm{~mm}$ & $141(32 \%)$ & $48(44 \%)$ & \\
\hline$>25 \mathrm{~mm}$ & $201(45 \%)$ & $32(30 \%)$ & \\
\hline \multicolumn{4}{|l|}{ No. positive lymph nodes $(n=536)$} \\
\hline 0 & $240(55 \%)$ & 79 (78\%) & \multirow[t]{3}{*}{$P<0.001^{c}$} \\
\hline $1-4$ & $119(27 \%)$ & $18(18 \%)$ & \\
\hline$>4$ & $76(17 \%)$ & $4(4 \%)$ & \\
\hline \multicolumn{4}{|l|}{ Tumour types } \\
\hline IDC & $323(70 \%)$ & $61(53 \%)$ & $P=0.001^{b}$ \\
\hline IDC and DCIS & $181(39 \%)$ & $49(43 \%)$ & $P=0.57^{b}$ \\
\hline DCIS (pure) & $62(14 \%)$ & $41(36 \%)$ & $P<0.001^{b}$ \\
\hline ILC & $79(17 \%)$ & $8(7 \%)$ & $P=0.008^{b}$ \\
\hline $\begin{array}{l}\text { Margin status }(\mathbf{n}=\mathbf{5 6 5}) \\
\text { Incomplete }(\leq 1 \mathrm{~mm})\end{array}$ & $68(15 \%)$ & $33(29 \%)$ & $P=0.001^{b}$ \\
\hline Lymphovascular invasion $(n=576)$ & $100(22 \%)$ & $14(12 \%)$ & $P=0.031^{b}$ \\
\hline ER positive $(n=531)$ & $347(81 \%)$ & $87(85 \%)$ & $P=0.37^{b}$ \\
\hline PR positive $(n=525)$ & $299(70 \%)$ & $78(78 \%)$ & $P=0.16^{b}$ \\
\hline $\begin{array}{l}\text { Her2 status }(\mathbf{n}=158) \\
{[3 \text { vs } 0,1,2]}\end{array}$ & $33(26 \%)$ & $10(30 \%)$ & $P=0.82^{b}$ \\
\hline LVI & $99(22 \%)$ & $14(12 \%)$ & $P=0.03$ \\
\hline Sentinel lymph node biopsy (SLNB) & $23(5 \%)$ & $9(8 \%)$ & $P=0.33^{b}$ \\
\hline
\end{tabular}

Axillary node surgery 


\section{elsevier_YEJSO_4342}

\begin{tabular}{|c|c|c|c|}
\hline Axillary node clearance (ANC) & $393(85 \%)$ & $67(58 \%)$ & \multirow[t]{3}{*}{$P<0.001^{b}$} \\
\hline Axillary node sampling (ANS) & $30(7 \%)$ & $20(17 \%)$ & \\
\hline None & $39(8 \%)$ & $28(24 \%)$ & \\
\hline Breast Reconstruction (Immediate or delayed) & $104(23 \%)$ & $113(98 \%)$ & $P<0.001^{b}$ \\
\hline \multicolumn{4}{|l|}{ Staging } \\
\hline Stage 0 & $32(7 \%)$ & $37(33 \%)$ & \multirow[t]{4}{*}{$P<0.001$} \\
\hline Stage 1 & $143(31 \%)$ & $39(34 \%)$ & \\
\hline Stage 2 & $180(40 \%)$ & $33(29 \%)$ & \\
\hline Stage 3 & $101(22 \%)$ & $4(4 \%)$ & \\
\hline \multicolumn{4}{|l|}{ Radiotherapy } \\
\hline Yes & $129(28 \%)$ & $12(11 \%)$ & \multirow[t]{2}{*}{$P<0.001^{b}$} \\
\hline No & $332(72 \%)$ & $101(89 \%)$ & \\
\hline \multicolumn{4}{|c|}{ Adjuvant or Neoadjuvant Chemotherapy (\% of chemotherapy) $(n=156)$} \\
\hline Adjuvant & $119(26 \%)$ & $37(33 \%)$ & $P=0.17^{b}$ \\
\hline Neoadjuvant & $34(7 \%)$ & $4(3 \%)$ & $P=0.21^{b}$ \\
\hline \multicolumn{4}{|l|}{ Hormone therapy } \\
\hline Yes & $335(73 \%)$ & $75(66 \%)$ & \multirow[t]{2}{*}{$P=0.21^{b}$} \\
\hline No & $125(27 \%)$ & $38(34 \%)$ & \\
\hline \multicolumn{4}{|l|}{ Recurrence: } \\
\hline Locoregional (local) & $27(19)$ & $9(8)$ & \\
\hline Five year & $5.6 \%(4.6 \%)$ & $5.7 \%(5.7 \%)$ & $P=0.73^{d}$ \\
\hline Eight year & $6.9 \%(5.0 \%)$ & $9.0 \%(7.9 \%)$ & $P=0.35^{d}$ \\
\hline
\end{tabular}

\section{a T-test.}

${ }^{b}$ Chi-square test.

' Linear trend.

${ }^{d}$ Log-Arank test.

\section{Surgical management}

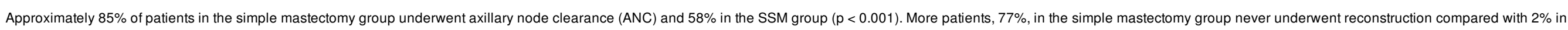
the SSM group $(p<0.001)$ (See Table 1).

\section{Adjuvant/neoadjuvant therapy}

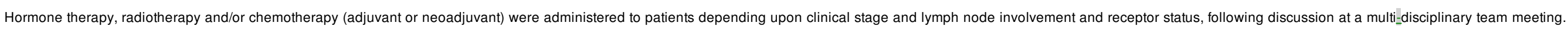




\section{elsevier_YEJSO_4342}

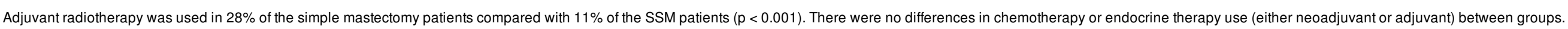

\section{Local, regional and systemic recurrences}

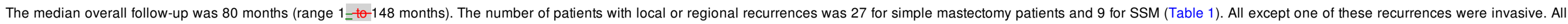
DCIS recurrences after mastectomy for pure DCIS presented as invasive recurrence.

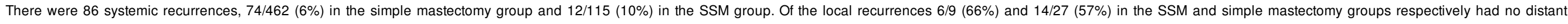
metastases.

Kaplan-Meier survival curves were used to calculate the proportion of patients with loco-regional recurrence at 5 and 8 -year intervals in each of the groups.

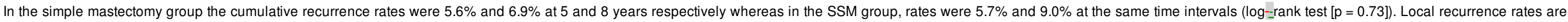
also given in Table 1.

\section{Predictors of recurrence and survival analysis}

Univariate analysis revealed patients with high grade tumours had a 2.61 times greater risk of local recurrence $(p=0.006)$ (Table 2). Involved nodes and incomplete margin clearance also increased local recurrence (Table 2).

Table 2 Factors predicting locoregional recurrence after SSM and simple mastectomy. Univariate Cox proportional hazards regression. Figures represent hazard ratios (95\% Cl); p-values.

\begin{tabular}{|c|c|c|c|}
\hline Characteristic & $\begin{array}{l}\text { Overall } \\
(n=577)\end{array}$ & $\begin{array}{c}\text { Simple } \\
(n=466)\end{array}$ & $\operatorname{SSM}(n=115)$ \\
\hline Mastectomy gro & & & \\
\hline SSM (vs simple) & $\begin{array}{l}1.14 \\
(0.53 \\
2.42) \\
P=0.74\end{array}$ & & \\
\hline Age (years) & $\begin{array}{l}0.99 \\
(0.97 \\
1.02) \\
p=0.56\end{array}$ & $\begin{array}{l}1.0(0.97 \\
1.03) \\
P=0.80\end{array}$ & $\begin{array}{l}0.92(0.84,0.99) \\
P=0.033\end{array}$ \\
\hline $\begin{array}{l}\text { Symptomatic } \\
\text { (vs screened) }\end{array}$ & $\begin{array}{l}1.31 \\
(0.64, \\
2.66) \\
P=0.46\end{array}$ & $\begin{array}{l}1.84 \\
(0.74 \\
4.56) \\
P=0.19\end{array}$ & $\begin{array}{l}0.63(0.17,2.35) \\
P=0.49\end{array}$ \\
\hline Grade 3 & $\begin{array}{l}2.61 \\
(1.28 \\
5.30) \\
\mathbf{P}=\mathbf{0 . 0 0 6}\end{array}$ & $\begin{array}{l}2.44 \\
(1.06 \\
5.57) \\
\mathbf{P}=\mathbf{0 . 0 3 5}\end{array}$ & $\begin{array}{l}2.98(0.74,11.9) \\
P=0.12\end{array}$ \\
\hline $\begin{array}{l}\text { ER positive } \\
(n=531)\end{array}$ & $\begin{array}{l}0.77 \\
(0.34 \\
1.77) \\
P=0.54\end{array}$ & $\begin{array}{l}0.75 \\
(0.30 \\
1.86) \\
P=0.54\end{array}$ & $\begin{array}{l}0.98(0.12,8.13) \\
P=0.98\end{array}$ \\
\hline $\begin{array}{l}\text { PR positive } \\
(n=525)\end{array}$ & $\begin{array}{l}0.70 \\
(0.34 \\
1.44) \\
P=0.34\end{array}$ & $\begin{array}{l}0.61 \\
(0.28 \\
1.33) \\
P=0.21\end{array}$ & $\begin{array}{l}1.72(0.21,14.3) \\
P=0.62\end{array}$ \\
\hline
\end{tabular}




\section{elsevier_YEJSO_4342}

\begin{tabular}{|l|l|l|l|} 
HER 2 status & 0.37 & 0.26 & $0.60(0.07,5.43)$ \\
( $=158)$ & $(0.09$, & $(0.03$, & $P=0.65$ \\
{$[$ 3 vs $0,1,2]$} & $1.63)$ & $2.02)$ & \\
& $P=0.19$ & $P=0.20$ &
\end{tabular}

\begin{tabular}{|c|c|c|c|}
\hline \multicolumn{4}{|c|}{ Tumour size $(n=548)$} \\
\hline $\begin{array}{l}<15 \mathrm{~mm} \\
15-25 \mathrm{~mm} \\
>25 \mathrm{~mm}\end{array}$ & $\begin{array}{l}1 \\
1.41 \\
(0.53, \\
3.75) \\
1.68 \\
(0.66, \\
4.27) \\
P=0.54\end{array}$ & $\begin{array}{l}1 \\
0.86 \\
(0.29, \\
2.56) \\
1.26 \\
(0.48, \\
3.28) \\
P=0.69\end{array}$ & $\begin{array}{l}1 \\
1 \\
1.28(0.30,5.34) \\
P=0.74^{a}\end{array}$ \\
\hline
\end{tabular}

\begin{tabular}{|l|l|l|l|}
\hline Staging & \multicolumn{3}{l|}{} \\
\hline Stage 0/1 & 1 & 1 & 1 \\
Stage 2 & 2.12 & 4.64 & $0.71(0.14,3.51)$ \\
Stage 3 & $(0.94$, & $(1.33$, & $3.23(0.39,26.9)$ \\
& $4.80)$ & $16.1)$ & $\mathrm{P}=0.45$ \\
& 3.79 & 7.26 & \\
& $(1.57$, & $(2.00$, & \\
& $9.15)$ & $26.4)$ & \\
& $\mathrm{P}=0.013$ & $\mathrm{P}=0.011$ & \\
\hline Lymphovascular & 2.66 & 3.26 & $1.02(0.13,8.12)$ \\
\hline invasion & $(1.35$, & $(1.52$, & $\mathrm{P}=0.99$ \\
& $5.25)$ & $6.96)$ & \\
\hline & $\mathrm{P}=0.005$ & $\mathrm{P}=0.002$ & \\
\hline
\end{tabular}

Positive lymph nodes

\begin{tabular}{|c|c|c|c|}
\hline $\begin{array}{l}0 \\
1-4 \\
>4\end{array}$ & $\begin{array}{l}1 \\
4.37 \\
(1.83, \\
10.4) \\
7.49 \\
(3.01, \\
18.7) \\
P<0.001\end{array}$ & $\begin{array}{l}1 \\
9.41 \\
(2.68,33) \\
14.5 \\
(3.98,53) \\
P<\mathbf{0 . 0 0 1}\end{array}$ & $\begin{array}{l}1 \\
0.89(0.10,7.63) \\
3.69(0.43,31.6) \\
P=0.47\end{array}$ \\
\hline $\begin{array}{l}\text { Any DCIS (pure } \\
\text { or with IDC } \\
\text { ( } n=571 \text { ) }\end{array}$ & $\begin{array}{l}0.84 \\
(0.43 \\
1.61) \\
P=0.60\end{array}$ & $\begin{array}{l}0.57 \\
(0.26 \\
1.23) \\
P=0.15\end{array}$ & b \\
\hline \multicolumn{4}{|l|}{ Margin status } \\
\hline $\begin{array}{l}\text { Complete } \\
\text { Incomplete } \\
(\leq 1 \mathrm{~mm})\end{array}$ & $\begin{array}{l}1 \\
2.92 \\
(1.48, \\
5.76) \\
P=0.002\end{array}$ & $\begin{array}{l}1 \\
2.86 \\
(1.25, \\
6.56) \\
P=0.013\end{array}$ & $\begin{array}{l}1 \\
3.34(0.90,12.4) \\
P=0.072\end{array}$ \\
\hline
\end{tabular}

Margin status by tumour type

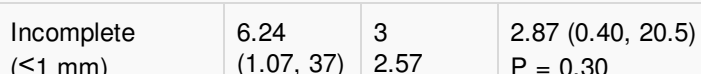

\begin{tabular}{l|l|l|l}
$(\leq 1 \mathrm{~mm})$ & $(1.07,37)$ & 2.57 & $\mathrm{P}=0.30$
\end{tabular} 


\section{elsevier_YEJSO_4342}

\begin{tabular}{|l|l|l|l|} 
Pure DCis & $\mathrm{P}=0.045$ & $(0.66$, & $2.62(0.44,15.7)$ \\
DCIS \& invasive & 2.82 & $9.94)$ & $\mathrm{P}=0.09$ \\
Invasive alone & $(1.00$, & $\mathrm{P}=0.17$ & 4 \\
& $7.92)$ & 2.72 & \\
& $\mathrm{P}=0.050$ & $(0.86$, & \\
& 2.56 & $8.58)$ & \\
& $(0.82$, & $\mathrm{P}=0.09$ & \\
& $7.96)$ & & \\
& $\mathrm{P}=0.11$ & &
\end{tabular}

C Only 1 recurrence hence no regression model convergence.

d No recurrence hence no regression model convergence.

a There were no recurrences in the $<15 \mathrm{~mm}$ subgroup, hence to obtain regression model convergence, the comparison ' $>25$ ' $v s$ ' $<=15$ ' was made.

${ }^{\mathrm{b}}$ No regression model convergence as no recurrences in the DCIS group (all 9 recurrences were in the non-DCIS group).

Amongst node negative patients, univariate analysis showed SSM was associated with a higher risk of relapse (despite lower lymphovascular invasion [LVI]) with a HR of $4.8(1.1-19.9 p=0.0033)$.

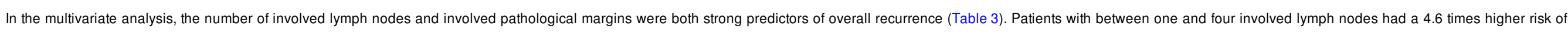

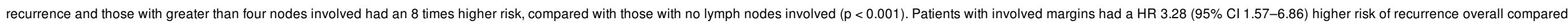
to those with no margin involvement (Table 3).

Table 3 Factors predicting locoregional recurrence after mastectomy (SSM and simple). Multivariable Cox proportional hazards regression. Figures represent hazard ratios (95\% Cl); p-values.

\begin{tabular}{|c|c|}
\hline & Overall hazard ratio \\
\hline \multicolumn{2}{|l|}{ Overall } \\
\hline \multicolumn{2}{|l|}{ Mastectomy type $^{a}$} \\
\hline $\begin{array}{l}\text { Simple } \\
\text { SSM }\end{array}$ & $\begin{array}{l}1 \\
1.05(0.43,2.56) \\
P=0.91\end{array}$ \\
\hline \multicolumn{2}{|c|}{ Positive lymph nodes } \\
\hline $\begin{array}{l}0 \\
1-4 \\
>4\end{array}$ & $\begin{array}{l}1 \\
4.64(1.93,11.2) \\
7.97(3.16,20.1) \\
\mathbf{P}<\mathbf{0 . 0 0 1}\end{array}$ \\
\hline \multicolumn{2}{|l|}{ Margin status } \\
\hline $\begin{array}{l}\text { Complete } \\
\text { Incomplete }(\leq 1 \mathrm{~mm})\end{array}$ & $\begin{array}{l}1 \\
3.28(1.57,6.86) \\
\mathbf{P}=\mathbf{0 . 0 0 2}\end{array}$ \\
\hline \multicolumn{2}{|c|}{ Lymph node negative patients } \\
\hline \multicolumn{2}{|l|}{ Mastectomy type } \\
\hline $\begin{array}{l}\text { Simple } \\
\text { SSM }\end{array}$ & $\begin{array}{l}1 \\
4.8(1.1,19.9) \\
\mathbf{p}=\mathbf{0 . 0 3 3}\end{array}$ \\
\hline
\end{tabular}

a After adjusting for positive lymph node status and margin status. 


\section{elsevier_YEJSO_4342}

\section{Factors predicting margin involvement}

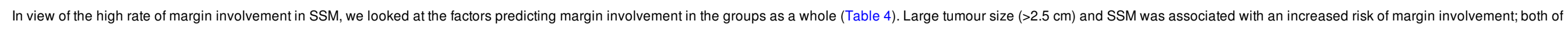
which remain significant in multi-variate analysis.

Table 4 Logistic regression analysis of factors associated with incomplete margins $(\leq 1 \mathrm{~mm})$. All patients $(n=577)$.

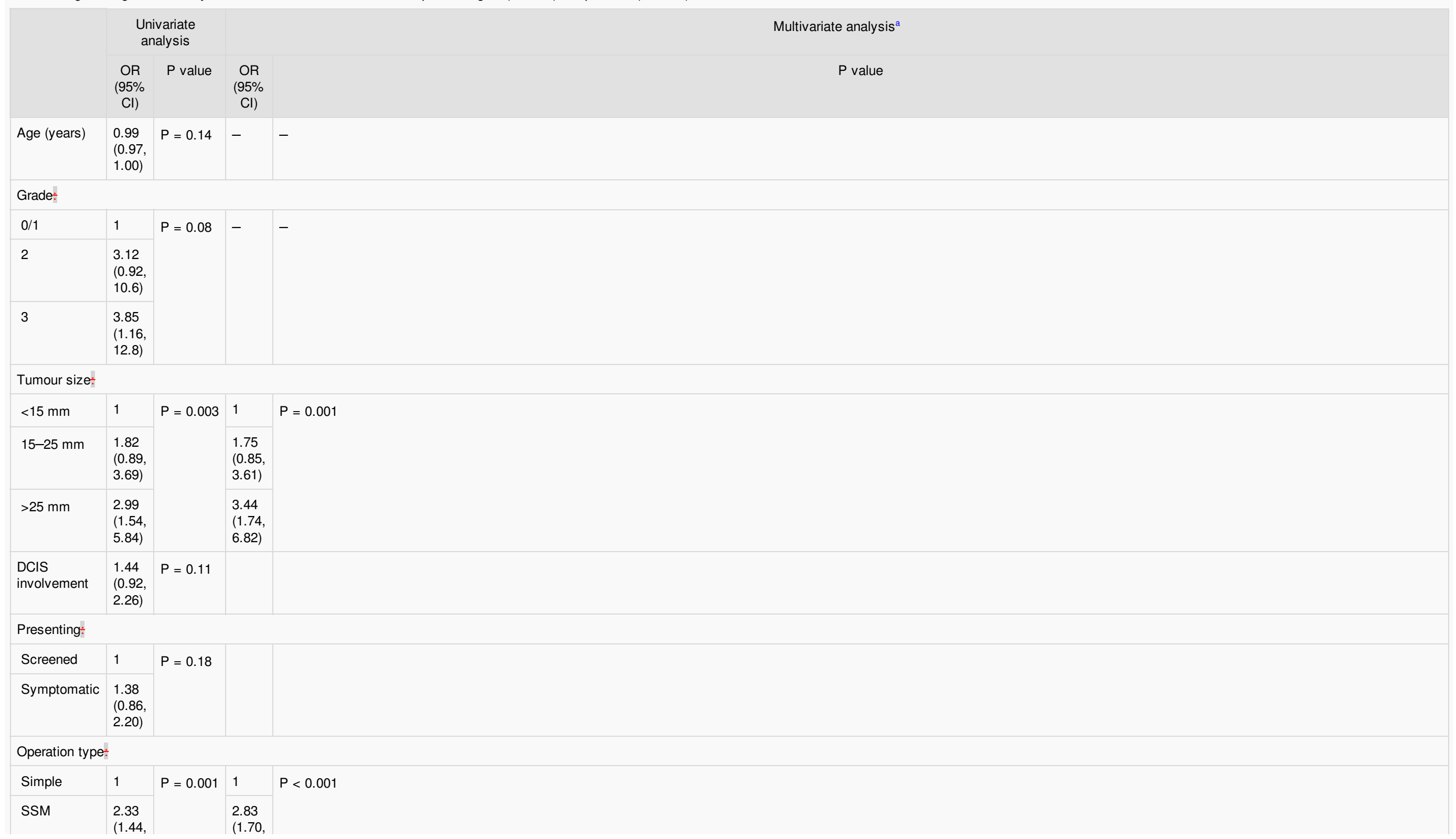




\section{elsevier_YEJSO_4342}

3.77)

4.73)

Tumour size, and operation type were all significantly associated with margin status when considered separately and multivariable analysis showed they were significant independent risk factors for incomplete margins.

a Significant independent associations.

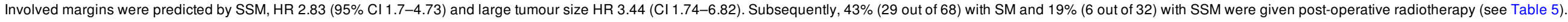

Table 5 Studies comparing local recurrence in SSM compared with simple mastecetomy.

\begin{tabular}{|c|c|c|c|c|c|c|c|c|}
\hline \multirow[t]{2}{*}{ First author } & \multirow{2}{*}{$\begin{array}{c}\text { Year of } \\
\text { publication }\end{array}$} & \multirow{2}{*}{$\begin{array}{l}\text { Years } \\
\text { covered }\end{array}$} & \multicolumn{2}{|c|}{ Patients } & \multicolumn{2}{|c|}{ Median follow up (months) } & \multicolumn{2}{|c|}{ Local recurrence (\%) } \\
\hline & & & SSM & NSSM & SSM & NSSM & SSM & NSSM \\
\hline Carlson $^{10}$ & 1997 & 1989-1994 & 327 & 188 & 15.6 & 32.4 & 4.8 & 9.5 \\
\hline Kroll11 & 1999 & $1986-1990$ & 114 & 40 & $>60$ & $>60$ & 7.0 & 7.5 \\
\hline Simmons $^{3}$ & 1999 & 1990-1998 & 77 & 154 & 15.6 & 32.4 & 3.9 & 3.2 \\
\hline Horiguchi ${ }^{12}$ & 2001 & 1993-1999 & 133 & 910 & 66 & 81 & 3.8 & 1.3 \\
\hline Greenway $^{13}$ & 2005 & 1989-2004 & 225 & 1022 & 49 & 49 & 7.1 & 5.4 \\
\hline Howard $^{14, a}$ & 2006 & $1987-2002$ & 34 & 395 & 59 & 59 & 0 & 3.8 \\
\hline Gerber $^{15}$ & 2009 & $1994-2000$ & 48 & 130 & 101 & 101 & 10.4 & 11.5 \\
\hline$Y i^{16}$ & 2011 & 2000-2005 & 799 & 1011 & 53 & 53 & 0.6 & 1.4 \\
\hline
\end{tabular}

a All patients underwent TRAM flap reconstructions.

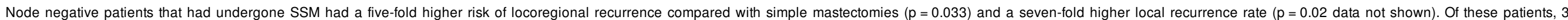
recurrences occurred in the 240 simple mastectomies and 5 in the 74 SSMs.

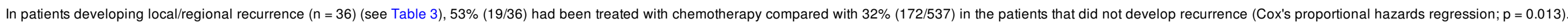

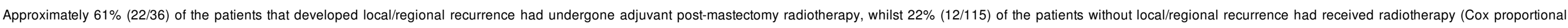

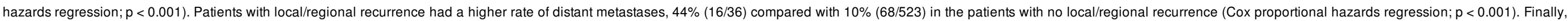
mortality was increased following local/regional recurrence at $61 \%(22 / 36)$, compared with $23 \%(122 / 541)$ in the patients with no recurrence (Cox proportional hazards regression; $p<0.001)$

\section{Discussion}

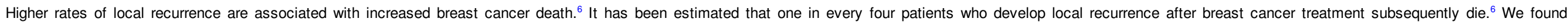
increased breast cancer mortality in patients who developed local recurrence. These patients may not have died, had their disease not recurred locally

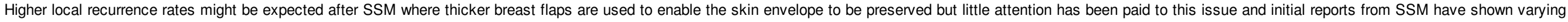

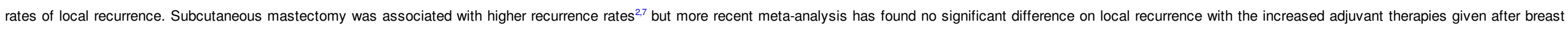
cancer surgery. ${ }^{3,8}$ This may relate to surgical technique ensuring skin overlying the cancer is sacrificed while still adhering to a skin preserving approach in the majority of cases in the meta-analysis.

Local recurrence rates in previous retrospective studies range from $0.6 \%$ to $10.4 \%$ in SSM and $1.3 \%$ to $11.5 \%$ after simple mastectomy (due to varying median follow-up ranging from 15.6 to 101 months).

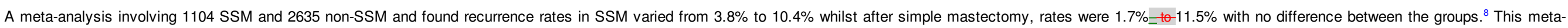

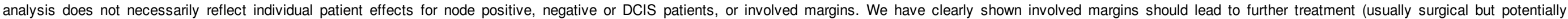


radiotherapy) to clear the margin.

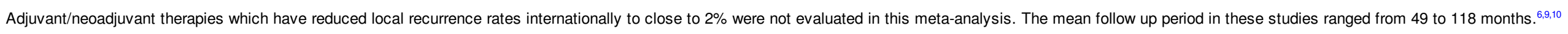

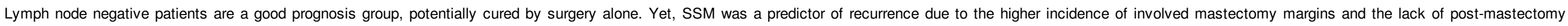

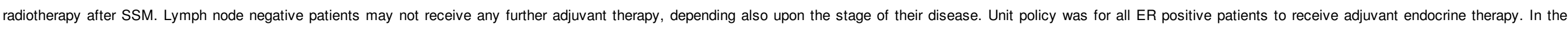

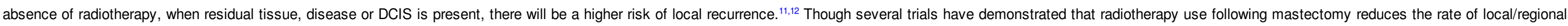

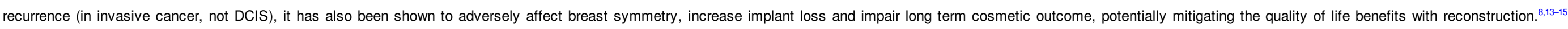

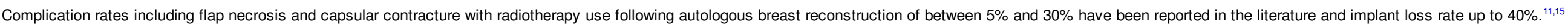

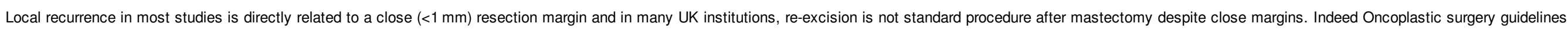
do not advise on management of involved margins after SSM. ${ }^{2,16-18}$

Our data indicates that failure to re-excise leads to an unacceptably high local recurrence rate after SSM and we have now changed our practice.

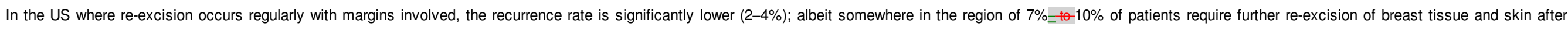
SSM. ${ }^{6,9}$

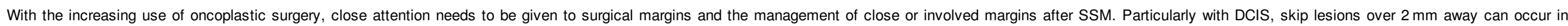
patients with widespread disease and SSM may be inappropriate..$^{15} \mathrm{An}$ alternative would be to remove more skin, particularly over the area of DCIS.

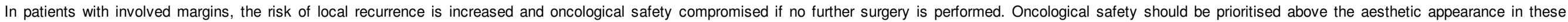
patients. We now ensure clear margins by re-excision of the margins after SSM if necessary, despite potential embarrassment to the Surgeon at explaining the issues to the patient.

Over $90 \%$ of all local recurrence after mastectomy occurs before five years have elapsed and late recurrence represents breast tissue left behind. ${ }^{3,6}$

Cutting thicker mastectomy flaps to avoid skin necrosis increases local recurrence and accounts for the increased rate of late local recurrence seen in the SSM group at eight years.

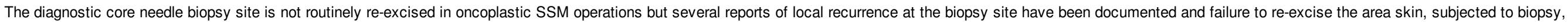
represents a risk of increasing local recurrence.

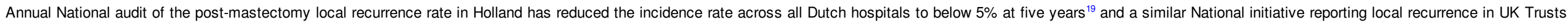
treating breast cancer is required to address ongoing problems (especially after SSM) with regard to local recurrence. Failure to audit and address these issues will lead to unnecessary breast cancer deaths in the UK.

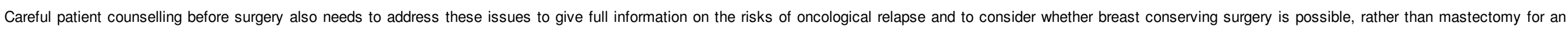
individual patient.

\section{Conflict of interest}

The authors have no conflict of interest to declare.

\section{Funding sources}

None.

\section{Uncited reference}

\section{References}




\section{elsevier_YEJSO_4342}

1

. B.A. Toth and P. Lappert, Modified skin incisions for mastectomy: the need for plastic surgical input in preoperative planning, Plast Reconstr Surg 87, 1991, 1048-1053.

2

. S.A. Robertson, J.E. Rusby and R.I. Cutress, Determinants of optimal mastectomy skin flap thickness, Br J Surg 101 (8), 2014, 899-911.

3

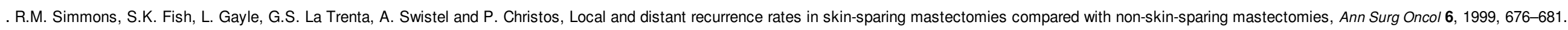

4

. R.Z. Torresan, C.C. dos Santos, H. Okamura and M. Alvarenga, Evaluation of residual glandular tissue after skin-sparing mastectomies, Ann Surg Oncol 12, 2005, 1037-1044.

5

. C.M. Ho, C.K. Mak, Y. Lau, W.Y. Cheung, M.C. Chan and W.K. Hung, Skin involvement in invasive breast carcinoma: safety of skin-sparing mastectomy, Ann Surg Oncol 10, $2003,102-107$.

6

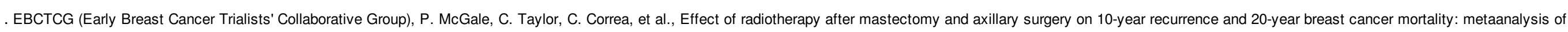

individual patient data for 8135 women in 22 randomised trials, Lancet 383 (9935), 2014, 2127-2135.

7

. B.V. Palmer, K.R. Mannur and W.B. Ross, Subcutaneous mastectomy with immediate reconstruction as treatment for early breast cancer, Br J Surg 79 (12), $1992,1309-1311$.

8

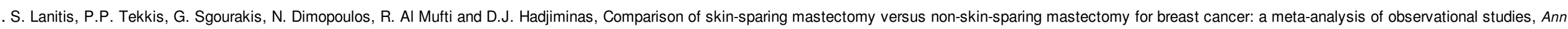
Surg 251, 2010, 632-639.

9

. J. Shen, J. Ellenhorn, D. Qian, D. Kulber and J. Aronowitz, Skin-sparing mastectomy: a survey based approach to defining standard of care, Am Surg 74, $2008,902-905$.

10

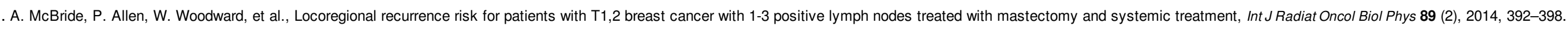

11

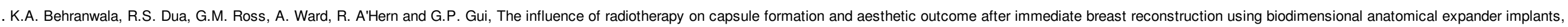
$J$ Plast Reconstr Aesthet Surg 59, 2006, 1043-1051.

12

. S.E. Singletary and G.L. Robb, Oncologic safety of skin-sparing mastectomy, Ann Surg Oncol 10, 2003, 95-97.

\section{3}

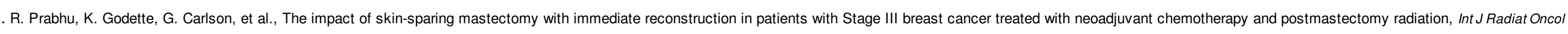
Biol Phys 82, 2012, e587-e593.

14

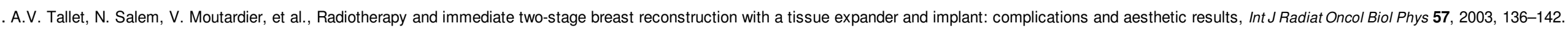




\section{elsevier_YEJSO_4342}

. N.P. Rowell, Are mastectomy resection margins of clinical relevance? A systematic review, Breast 19, 2010, 14-22.

16

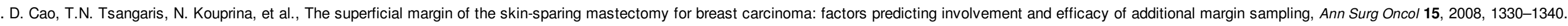

17

. G.W. Carlson, T.M. Styblo, R.H. Lyles, J. Bostwick, D.R. Murray and C.A. Staley, Local recurrence after skin-sparing mastectomy: tumor biology or surgical conservatism?, Ann Surg Oncol 10, $2003,108-112$.

18

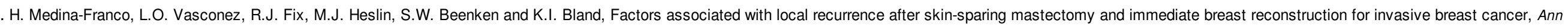
Surg 235, 2002, 814-819.

19

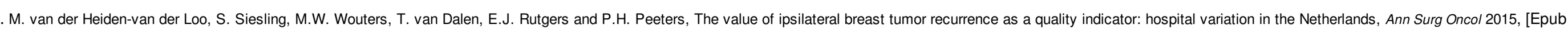
ahead of print].

20

. J.L. Uriburu, H.D. Vuoto, L. Cogorno, et al., Local recurrence of breast cancer after skin-sparing mastectomy following core needle biopsy: case reports and review of the literature, Breast J 12 (3), 2015 , 194-198.

\section{Queries and Answers}

Query: Please check the inserted keywords, and correct if necessary.

Answer: Satisfied with correction, thank you.

Query: Please note that Table 5 was not cited in the text. Please check that the citation suggested is in the appropriate place, and correct if necessary.

Answer: This table is to be left out of the main manuscript, please. It was included for use as a supplementary table.

Query: The significance symbol P, has been represented by both P and p, and needs to be consistent throughout the article. Please check and amend as necessary.

Answer: Apologies. Please amend to a small ' $p$ ' throughout the manuscript.

Query: Please check the 'Conflict of interest' and 'Funding sources' section, and correct if necessary.

Answer: Both sections are correct, thank you.

Query: Please provide the volume number or issue number or page range or article number for the bibliography in Ref. 19.

Answer: Please update the publication details to Ann Surg Oncol. 2015 Dec;22 Suppl 3:S522-8.

Query: Please check the layout of Tables $1-4$, and correct if necessary.

Answer: All appear to be correct, thank you.

Query: Please provide a definition for the significance of bold in the Tables 1 and 2.

Answer: Please remove the 'bolding' and replace with standard text format.

Query: Please note that, figures are not present in the article. Hence, please check the representation of figures in the caption of Tables 1 and 2, and correct if necessary.

Answer: We cannot find any errors in the table captions. Please can you clarify what is meant by this query?

Query: Table footnote 'c' and ' $d$ ' for the Table 2 were provided, but not cited in the table. Please provide a citation for them, and correct as necessary. 


\section{elsevier_YEJSO_4342}

Answer: Please remove footnotes $C$ and $D$.

Query: Please note that the citations present in the first column of Table 5 have not been matched with the author name before that reference citation. Kindly check, and correct if necessary.

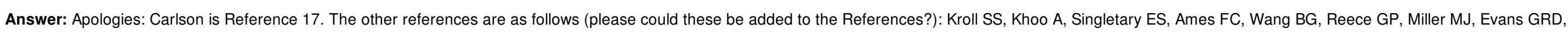

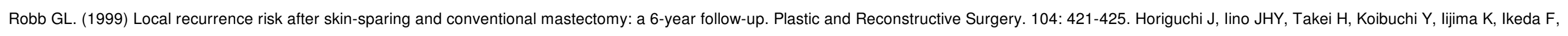

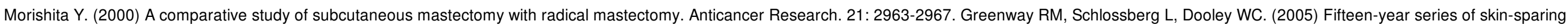

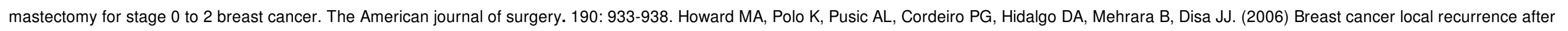

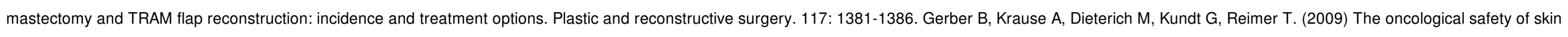

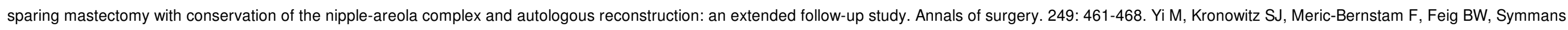
WF, Lucci A, Hunt KK. (2011) Local, regional, and systemic recurrence rates in patients undergoing skin-sparing mastectomy compared with conventional mastectomy. Cancer. 117: 916-924.

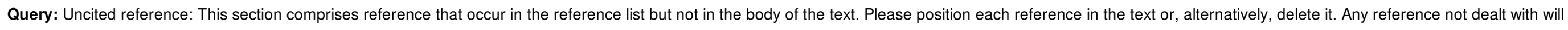
be retained in this section. Thank you.

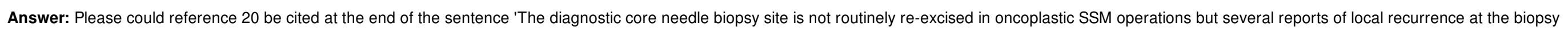
site have been documented and failure to re-excise the area skin, subjected to biopsy, represents a risk of increasing local recurrence.' (in the Discussion section).

Query: Please confirm that given names and surnames have been identified correctly.

Answer: Yes thank you.

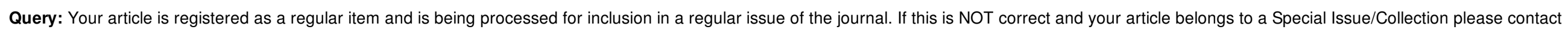
a.chadburn@elsevier.com immediately prior to returning your corrections.

Answer: This is correct, thank you. 